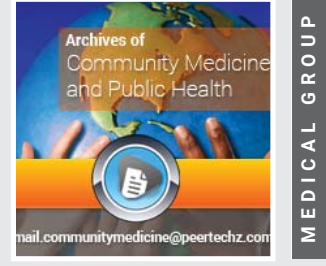

\section{The Prevalence of Left}

\section{ventricular hypertrophy as a}

\section{feature of Hypertensive Heart}

\section{Disease in A Tertiary Hospital in}

\section{Tanzania: An Echocardiographic Registry Study}

\author{
Henry Anselmo Mayala*, Delila Kimambo, Engerasiya Julius \\ Kifai, Siza J Ngomero, Abuu Abdallah Dalidali, Paskal \\ Elia Kondi, Gerson Imael Mpondo, Saada A Salum, Pedro \\ Pallangyo and Mohamed Janabi
}

Jakaya Kikwete Cardiac Institute, P. O. Box 65141 Dar es Salaam, West Upanga, Kalenga Street, Ilala District, Dar es Salaam Region, Tanzania
Received: 20 October, 2020

Accepted: 27 November, 2020

Published: 28 November, 2020

*Corresponding author: Henry Anselmo Mayala Jakaya Kikwete Cardiac Institute, P. O. Box 65141 Dar es Salaam, West Upanga, Kalenga Street, Ilala District, Dar es Salaam Region, Tanzania, Tel: +255-22-2152392, +255 755144363, +255-762400465;

Email: mayalahenry29@gmail.com

Keywords: Echocardiography; Hypertensive heart disease; Dilated cardiomyopathy; Rheumatic heart disease; Left ventricular hypertrophy

https://www.peertechz.com

Check for updates

\title{
Abstract
}

Objective: Echocardiography is a useful noninvasive tool for the diagnosis and evaluation of cardiac diseases. This study aims to share our 3 months' experience in our echo lab as well as the scope of clinical cases diagnosed at our institute.

Methods: This is a cross-sectional observational study conducted at the Jakaya Kikwete Cardiac Institute, in Dar-es-salaam, Tanzania between February 2020 to May 2020. This was performed with the Siemens Accuson S-300 machine using two dimensional, M-mode, colour flow and tissue Doppler protocols.

Results: 1992 consecutive patients, 857 males and 1135 females, were examined during the study period. Their ages ranged from 27 to 84 years within a mean of $55 \pm 13$ years. $1236(62 \%)$ had left ventricular hypertrophy and diastolic dysfunction as features of hypertensive heart disease, 145 ( $7.2 \%)$ had dilated cardiomyopathy, 87 (4.4\%) had ischemic cardiomyopathy, 54 (2.7\%) had Rheumatic heart disease, also we had 450 (22.6\%) who had a normal echo.

Conclusion: Hypertensive heart disease was the immense echocardiographic diagnosis at our institute and is the most significant non-communicable diseases responsible for increased morbidity and mortality among our patients in Tanzania. Furthermore, a lot of effort needs to be done to increase awareness, prevention, treatment and drug compliance for hypertension to combat the complications.

\section{Abbreviations}

LVH: Left Ventricular Hypertrophy; JKCI: Jakaya Kikwete Cardiac Institute

\section{Introduction}

Hypertension is a highly prevalent disease worldwide with significant morbidity and mortality. There are several predisposing factors for hypertension which include age, genetics, environmental factors, obesity and sedentary lifestyle [1].

Hypertensive heart disease is characterized by Left Ventricular Hypertrophy (LVH) in absence of any other cause apart from systemic arterial hypertension. Furthermore, it can 
also be described as structural cardiac disorder accompanied by concentric LVH, diastolic and either systolic dysfunction [2-5].

Left ventricular hypertrophy and diastolic dysfunction tend to develop in patients with long-standing hypertension. Left ventricular hypertrophy is a crucial prognostic indicator in hypertensive heart disease patients signifying the role of those who need more aggressive blood pressure control [2].

Assessment of left ventricular hypertrophy by echo is crucial especially in maximizing treatment with hypertensive, whereby treatment with antihypertensive has shown to improve diastolic function and cardiovascular outcomes [3].

Since its inauguration in 2015 Jakaya Kikwete Cardiac Institute, more than 20,000 echocardiograms were done each year, with an average of 1800 echocardiograms every month, in 2016 the institute decided to have a dedicated echo lab.

This study aims to report our findings and experience with the procedure for 3 months as well as the spectrum of clinical cases diagnosed at our institute.

\section{Methodology}

Study population: We recruited 1992 consecutive patients attending Jakaya Kikwete Cardiac Institute outpatient clinic.

Study design: A cross-sectional prospective observational study.

Study Objective: the intension of this study was to share and report the 3 months' experience in our echo lab as well as the scope of clinical cases diagnosed at our institute.

Clinical evaluation: We obtained demographic profile data such as age, sex, and indication for the echo from the participants. Before echo, a referring physician obtains detailed history, physical examination, ECG and Chest x-ray.

Echocardiography: This was performed with the Siemens Acuson-X 300 using 2D, colour flow and tissue Doppler protocol. Echocardiography was performed on each patient in the left lateral decubitus position. All measurements were made according to the American Society of Echocardiography leadingedge to leading-edge convention protocol. Echocardiography examination was performed in the parasternal long axis, short axis, apical 4 chamber and occasionally subcostal views. Left ventricular measurements were obtained at end-diastole and end-systole in the parasternal long-axis view. Three experienced cardiologists performed all the echocardiographic interpretations. All echocardiographic diagnoses were based on standard criteria [6].

Ethical clearance: This study was approved by the ethics committee of Jakaya Kikwete Cardiac Institute. This study was conducted according to the revised declaration of Helsinki concerning biomedical research in using patient information.

\section{Results}

1992 consecutive patients were recruited during the study period, whereby 857 were males and 1135 were females. Their ages ranged from 27 to 84 years with a mean of $55 \pm 13$ year. The clinical indications for echocardiography are summarized in Table 1. The most common clinical indications for patients being referred for echocardiography were: Hypertensive heart disease 996 (50\%); Dilated cardiomyopathy 300 (15\%); chest discomfort $250(12.5 \%)$; congestive heart failure 100 (5\%); and rheumatic heart disease $100(5 \%)$.

The diagnoses made at echocardiography are shown in Table 2. The most prevalent diagnoses were hypertensive heart disease (1236 patient; 62\%) and dilated cardiomyopathy (145 patients; $7 \%$ ) while 450 patients (22.6\%) had normal echo study. Hypertensive heart disease patients were prevalent among patients aged $50-84$ years $(70 \%, 865$ patients) vs those aged 27-49 years (30\%, 371 patients).

Evaluation of data for distinctive analysis showed that of the 996 patients who came for assessment for hypertensive heart disease, 946 patients were confirmed with a yield of $95 \%$ while the others had a normal study. Dilated cardiomyopathy was confirmed in $201(67 \%)$ of the 300 patients and ischemic heart disease and rheumatic in $95(95 \%)$ out of 100 and $40(40 \%)$ out of 100 respectively (Table 3 ).

Furthermore, out of 450 normal echo studies 66\% (300) were from outside JKCI but in Dar-es-salaam region, 6\% (25) from within JKCI and 28\% (125) from other regions.

Table 1: Showing indications for Echocardiography.

\begin{tabular}{|c|c|c|}
\hline Clinical Indication & Total $\boldsymbol{n}$ of Request & $\%$ \\
\hline HHD & 996 & 50 \\
\hline DCM & 300 & 15 \\
\hline Chest discomfort & 250 & 12.5 \\
\hline IHD & 100 & 5 \\
\hline CHF & 100 & 5 \\
\hline RHD & 100 & 5 \\
\hline Prosthetic Heart Valve & 50 & 2.5 \\
\hline Chemotherapy & 50 & 2.5 \\
\hline Arrhythmia & 20 & 1 \\
\hline Cardiomegaly on CXR & 26 & 1.3 \\
\hline
\end{tabular}

HHD: Hypertensive Heart Disease; DCM: Dilated Cardiomyopathy; IHD: Ischemic Cardiomyopathy; CHD: Congestive Heart Failure; RHD: Rheumatic Heart Disease; CXR: Chest X-ray

Table 2: Echocardiographic Diagnosis.

\begin{tabular}{|c|c|c|}
\hline Diagnosis & Total no. of patients & $\%$ \\
\hline Hypertensive heart disease & 1236 & 62 \\
\hline Normal echo studies & 450 & 22.6 \\
\hline Dilated cardiomyopathy & 145 & 7.2 \\
\hline Ischemic cardiomyopathy & 87 & 4.4 \\
\hline Rheumatic heart disease & 54 & 2.7 \\
\hline Cor-pulmonale & 12 & 0.6 \\
\hline Pulmonary hypertension & 5 & 0.3 \\
\hline Congenital (vsd and asd) & 2 & 0.1 \\
\hline Hypertrophic cardiomyopathy & 1 & 0.05 \\
\hline
\end{tabular}

Citation: Mayala HA, Kimambo D, Kifai EJ, Ngomero SJ, Dalidali AA, et al. (2020) The Prevalence of Left ventricular hypertrophy as a feature of Hypertensive Heart Disease in A Tertiary Hospital in Tanzania: An Echocardiographic Registry Study. Arch Community Med Public Health 6(2): 270-273. DOI: https://dx.doi.org/10.17352/2455-5479.000119 
Table 3: Confirmation rate of clinical diagnosis at echocardiography. \begin{tabular}{|l|l|l} 
Clinical diagnosis & Total request $(n)$ & Confirmed by echo
\end{tabular} $\%$ $\mathrm{HHD}$

996

946

DCM

300

201

IHD

100

40

67

RHD

100

98

\section{Discussion}

The findings from our registry so far have shown hypertension as the leading indication for requesting echocardiography while hypertensive heart disease was the prevalent diagnosis. Dilated cardiomyopathy was the next common diagnosis after hypertensive heart disease. Our results were similar to previous studies done in Tanzania revealing the surge of hypertensive heart disease in our country [7-10].

Our results are kindred with researches done in other African countries particularly the one published in 2012 by Odia, et al. in which they scanned 234 patients, whereby $53 \%$ had hypertensive heart disease, $9 \%$ had rheumatic heart disease and $6 \%$ had dilated cardiomyopathy this study was done in Nigeria. Our study followed the same pattern nevertheless dilated cardiomyopathy was more common than rheumatic heart diseases and more of our participants $(62 \%)$ had hypertensive heart disease in contrast with $52 \%$ in Odia, et al. study [11]. This may be elaborated by the surge of hypertension in our society and the increment in the number of hypertensive patients being referred for echocardiography. Furthermore, another clarification for the high prevalence of hypertensive heart disease maybe because of the poor control of hypertension that has resulted in structural changes of left ventricular hypertrophy. Hypertensive heart disease in this study was common in both genders (male and female) though slightly more in females depicting the rise in prevalence of hypertensive heart disease in Tanzanian Women, our research in keeps with Odia, et al. findings [11]. 90\% (78) of the ischemic heart disease patients were men insinuating that the male gender is a risk factor for ischemic heart disease.

In previous studies done in different regions of Tanzania, which was not echocardiographic registry programs the prevalence of hypertension was $8 \%, 28 \%, 36 \%$ respectively $[1,8,9$,$] in mostly were done in upcountry, but in 2016$ there was another study done in Dar-es-salaam city in which the prevalence of hypertension was $61.5 \%$ [12-15].

As previously explained in prior researches done, the prevalence of hypertension is increasing as the years goes by due to urbanization, poor drug compliance and increasing awareness of non-communicable diseases causing more people to come to do a check-up, all this necessitate the opportunity for a large multi-centre study, to get the real picture. Moreover, through our observation study, we see a chance to educate the community about hypertension, its overview and complications, the importance of drug adherence and health education about Non-Communicable Diseases (NCD's) in general.

The escalated use of echocardiography in our echo lab may contemplate the increased physician's cognizance of the need for a complete evaluation of cardiovascular patients to guide treatment using best practices. There is also a need for judicious use of this implement by the initiation of specific indications for referral to avoid unnecessary investigations and waste of patients resources.

\section{Limitation}

Our study limitation was the inclusion of only participants who attended to our cardiac institute.

The participants in the study could still diverge from the prevailing population by being more or less health-conscious and having severe disease.

\section{Conclusion}

Echocardiography is a practical implementation in the assessment and identification of cardiac diseases and as divulged in this study, hypertension and hypertensive heart disease comprise the largest clinical indication and echocardiographic diagnosis at our institute. The commonness of hypertensive heart disease emphasizes the requirement for more belligerent control of hypertension among our patients with more urgency on patient education to improve compliance to medication and a healthy lifestyle.

\section{Declarations}

Ethical approval and consent to participate: The clinical protocol and the informed consent forms were approved by the Research ethics committee of JKCI. All patients read and signed the published. informed consent. This observational echo registry study was conducted according to the revised declaration of Helsinki concerning biomedical research in using patient information. All authors agreed for this manuscript to be published.

\section{Availability of data and materials}

Data and materials are available upon request to the authors

\section{Authors contribution}

Drafting of manuscript was done by: HAM, DK, EJK, S, ABD, PEK, GIM, PD. Critical revision and correction were done by: MJ. All authors read and approved the final manuscript.

\section{Acknowledgements}

We thank the Staffs and management of Jakaya Kikwete Cardiac Institute for granting permission to conduct this study. And we thank all participants who participated in the study.

\section{References}

1. Muhamedhussein MS, Nagri ZI, Manji KP (2016) Prevalence, Risk factors, Awareness, and Treatment and Control of Hypertension in Mafia Island, Tanzania. Hindawi, International Journal of Hypertension 2016: 1281384. Link: https://bit.ly/3q9fzqe

2. Lalande S, Johnson B (2008) Diastolic dysfunction: A link between hypertension and heart failure. Drugs Today 44: 500-513. Link: https://bit.ly/36cW4pa 
3. Mozdzan M, Drabik KW, Kurpesa M, Trzos E, Rechciński T, et al. (2013) Echocardiographic indices of left ventricular hypertrophy and diastolic dysfunction in hypertensive patients with preserved LVEF classified as dippers and non-dippers. Arch Med Sci 9: 268-275. Link: https://bit.ly/2JnP4Ne

4. Gaziano JM (2008) Global Burden of Cardiovascular Disease. Braunwald's Heart Disease: A textbook of cardiovascular medicine. $8^{\text {th }}$ ed. Saunders Inc $1-21$

5. Sahn DJ, DeMaria A, Kisslo J, Weyman A (1978) Recommendations regarding quantification in $\mathrm{m}$-mode echocardiography: results of a survey of echocardiographic measurements. Circulation 56: 1072-1083. Link: https://bit.ly/2V5Lppl

6. Galson SW, Stanifer JW, Herz JT, Temu G, Thielman N, et al. (2019) The burden of hypertension in the emergency department and linkage to care: A prospective cohort study in Tanzania. Plos One 14: e0211287. Link: https://bit.ly/3mf9Mgu

7. Galson SW, Staton CA, Karia F, Lunyera J, Patel UD, et al. (2017) Epidemiology of hypertension in Northern Tanzania: a community-based mixed-methods study. BMJ Open 7: e018829. Link: https://bit.ly/3q6d6gt

8. Mosha NR, Mahande M, Juma A, Mboya I, Peck R, et al. (2017) Prevalence, awareness and factors associated with hypertension in North West Tanzania. Global Health Action 10: 132121279. Link: https://bit.ly/3m8qoXn

9. Isangula K, Meda JR (2017) The burden of hypertension in the rural and urban populations of Tanzania: A review of Trends, impacts and response. Tanzania Journal of Health Sciences 1: 41-52. Link: https://bit.ly/2JdivRT

10. James OO, Efosa JD, Romokeme AM, Zuobemi A, Sotonye DM (2012) Dominance of Hypertensive Heart Disease in a Tertiary Hospital in Southern Nigeria: An Echocardiographic Study. Ethn Dis 22: 136-139. Link: https://bit.ly/33p6m3o

11. Janardhanan R, Kramer CM (2011) Imaging in hypertensive disease. Expert Rev Cardiovasc Ther 9: 199-209. Link: https://bit.ly/2VbkzfQ

12. Pedro $P$, Pauline $P$, Peter $K$, Mayala $H$, Swai $N$, et al. (2016) A community based study on prevalence and correlates of erectile dysfunction among Kinondoni District Residents, Dar es salaam, Tanzania. Reprod Health 13: 140. Link: https://bit.ly/36cAINP

13. Muhihi AJ, Njelekela MA, Mpembeni RNM, Muhihi BG, Anaeli A, et al. (2018) elevated blood pressure among primary school children in Dar-essalaam, Tanzania: Prevalence and risk factors. BMC Pediatr 18: 54. Link: https://bit.ly/3lgrVt4

14. Zack RM, Irema K, Kazonda P, Leyna GH, Liu E, et al. (2016) Determinants of high blood pressure and barriers to diagnosis and treatment in Dar-es-salaam, Tanzania. Journal of Hypertension 34: 2353-2364. Link: https://bit.ly/33okytv

15. The American Society of Echocardiography Recommendations for Cardiac Chamber Quantification in Adults: A Quick Reference Guide from the Ase Workflow and Lab Management Task Force. Link: https://bit.ly/39r8wnf

\section{Discover a bigger Impact and Visibility of your article publication with}

\section{Peertechz Publications}

Highlights

* Signatory publisher of ORCID

* Signatory Publisher of DORA (San Francisco Declaration on Research Assessment)

* Articles archived in worlds' renowned service providers such as Portico, CNKI, AGRIS, TDNet, Base (Bielefeld University Library), CrossRef, Scilit, J-Gate etc.

* Journals indexed in ICMJE, SHERPA/ROMEO, Google Scholar etc.

* OAI-PMH (Open Archives Initiative Protocol for Metadata Harvesting)

* Dedicated Editorial Board for every journa

Accurate and rapid peer-review process

Increased citations of published articles through promotions

* Reduced timeline for article publication

Submit your articles and experience a new surge in publication services (https://www.peertechz.com/submission).

Peertechz journals wishes everlasting success in your every endeavours.

Copyright: @ 2020 Mayala HA, et al. This is an open-access article distributed under the terms of the Creative Commons Attribution License, which permits unrestricted use, distribution, and reproduction in any medium, provided the original author and source are credited. 